\title{
Initial maturity model for information governance arrangements in organizations
}

\author{
Diogo Proença, Ricardo Vieira, José Borbinha \\ INESC-ID, Instituto Superior Tecnico, Universidade de Lisboa \\ Rua Alves Redol n 9, Lisbon, Portugal \\ diogo.proenca@tecnico.ulisboa.pt,rjcv@tecnico.ulisboa.pt, jlb@tecnico.ulisboa.pt \\ Clive Billenness \\ University of Portsmouth \\ 2 Winston Churchill Avenue, Portsmouth, Hampshire, UK \\ clive@fp7experts.eu
}

\section{Introduction}

The workshop is based on the research work undertaken in the EC Project E$\mathrm{ARK}^{1}$ [620998] as published in public Project Deliverable D7.2, submitted to the EC on 1 August 2015.

\section{Workshop outline}

The purpose of the workshop is to introduce delegates to the background and principles of the Maturity Model which measures the effectiveness of the Information Governance Arrangements within an organisation - which are essential to support the archiving of electronic data. This will be followed by a detailed inspection of the evaluation model and an examination of the questions.

Delegates will be invited to take a copy of the model back to their own organisation and complete it to assess their own current maturity level in a number of areas and so develop an action plan to address any shortcomings identified.

Delegates will also be invited to submit their responses anonymously to a central database, thereby contributing to a facility for organisations to benchmark themselves again comparable bodies and measure their progress.

The workshop is intended to be highly interactive and is intended for all involved in the archiving of electronic data or preparing for such activities.

\footnotetext{
${ }^{1}$ E-ARK (European Archival Records and Knowledge Preservation) Project, http://www. eark-project.com/
} 